\title{
Using Predictive Scoring Systems for Asthma Exacerbations Could Help Safely Conserve Resources \\ During the COVID-19 Pandemic
}

\author{
Michael D Davis
}

A number of clinical scoring systems for asthma management have been described in the literature over the last four decades. ${ }^{1}$ These systems are largely used to index severity of disease, quantify changes in disease status, and predict outcomes. Resulting scores can then be used to triage patients and guide therapy. Although they can be very useful tools, scoring systems are not perfect. To create a scoring system, one must create generalized categories of symptoms and ranges of severity. Because asthma is a heterogeneous disease with many causes (and treatments), it is difficult to create a broad system to appropriately score this disease. Moreover, most asthma scoring systems are designed for adult patients and must be modified for pediatric patients.

In this issue of the Journal, Miller et $\mathrm{al}^{2}$ evaluated the modified Pulmonary Index Score (mPIS), which is a version of the Pulmonary Index that is modified to adjust vital sign parameters for the age of pediatric patients. They have reported that the MPIS at the onset of admission was predictive of length of stay (LOS) in the hospital, LOS in the ICU, and duration of continuous albuterol therapy, similar to findings of previous studies. Several groups at different centers have published findings that the MPIS is predictive of LOS, correlates with disease severity, and is reproducible across groups of health care professionals, supporting the notion that the mPIS may, indeed, be a useful tool when treating pediatric patients experiencing asthma exacerbations. ${ }^{2-5}$ However, the observations from these trials may not replicate well at other centers with different patient demographics and asthma exacerbation management

This is the author's version of the work published in final form as:

Davis, M. D. (2020). Using Predictive Scoring Systems for Asthma Exacerbations Could Help Safely Conserve Resources During the COVID-19 Pandemic. Respiratory Care, 65(9), 1413. https://doi.org/10.4187/respcare.08369 
protocols. More prospective, multicenter studies comparing the mPIS to other scoring systems are warranted.

Miller et $\mathrm{al}^{2}$ also reported that admission mPIS predicted the need for high-flow nasal cannula and heliox therapy, which generally require an in-patient setting to administer. These findings may be of particular value during the current SARS-CoV-2 pandemic. As many hospitals throughout the world experience periods of census overload from respiratory patients, canonical triage criteria are not effective. ${ }^{6}$ We are already seeing an increase in remote treatment and monitoring of respiratory patients worldwide in response to the pandemic. ${ }^{7}$ Objective metrics that can predict disease severity, course, and LOS could assist in determining which patients should be admitted and which can be managed effectively as outpatients to protect them from exposure to SARS-CoV-2 and to conserve in-patient resources.

Further studies evaluating the usefulness of MPIS for predicting outcomes in pediatric patients who are experiencing asthma exacerbations would also be beneficial during these times. These benefits could easily expand beyond the pediatric asthma population to all age groups and other patients with exacerbation-prone respiratory diseases, such as cystic fibrosis and COPD. These and other predictive tools could help us safely conserve respiratory health care resources and give the best care we can to our patients as we continue to adapt to the pandemic.

\section{Footnotes}

- Correspondence: Michael D Davis RRT PhD. E-mail: mdd1@iu.edu

- Dr Davis is funded by the NIH and the Indiana Clinical and Translational Science Institute and also a patent holder for Optate and co-founder of Airbase Breathing Company. 


\section{References}

1. van der Windt DA, Nagelkerke AF, Bouter LM, Dankert-Roelse JE, Veerman AJ. Clinical scores for acute asthma in pre-school children. A review of the literature. J Clin Epidemiol 1994;47(6):635-646.

2. Miller AG, Haynes KE, Gates RM, Zimmerman KO, Bartlett KW, McLean HS, et al. Initial modified pulmonary index score predicts hospital length of stay for asthma subjects admitted to the pediatric intensive care unit. Respir Care 2020;65(9):1227-1232.

3. Carroll CL, Sekaran AK, Lerer TJ, Schramm CM. A modified pulmonary index score with predictive value for pediatric asthma exacerbations. Ann Allergy Asthma Immunol 2005;94(3):355-359.

4. Koga T, Tokuyama K, Itano A, Morita E, Ueda Y, Katsunuma T. Usefulness of modified Pulmonary Index Score (mPIS) as a quantitative tool for the evaluation of severe acute exacerbation in asthmatic children. Allergol Int 2015;64(2):139-144.

5. Maekawa T, Oba MS, Katsunuma T, Ishiguro A, Ohya Y, Nakamura H. Modified pulmonary index score was sufficiently reliable to assess the severity of acute asthma exacerbations in children. Allergol Int 2014;63(4):603-607.

6. Comelli I, Scioscioli F, Cervellin G. Impact of the COVID-19 epidemic on census, organization and activity of a large urban emergency department. Acta Biomed 2020;91(2):45-49.

7. Shaker MS, Oppenheimer J, Grayson M, Stukus D, Hartog N, Hsieh EWY, et al. COVID-19: pandemic contingency planning for the allergy and immunology clinic. J Allergy Clin Immunol Pract 2020;8(5):1477-1488. 